\title{
Two-Dimensional Electronic Spectroscopy Reveals Dynamics and Mechanisms of Solvent-Driven Inertial Relaxation in Polar BODIPY Dyes
}

\author{
Luca Bolzonello, ${ }^{\dagger}$ Annalisa Polo, ${ }^{\dagger}$ A Andrea Volpato, ${ }^{\dagger}$ Elena Meneghin, ${ }^{\dagger}$ Massimiliano Cordaro, ${ }^{\ddagger}, \mathbb{I}$
}

Mariachiara Trapani, ${ }^{\S}$ Mariagrazia Fortino,, Alfonso Pedone, ${ }^{\| \odot}$ Maria Angela Castriciano, ${ }^{\perp}$

and Elisabetta Collini* ${ }^{\dagger \dagger}$

\begin{abstract}
${ }^{\dagger}$ Dipartimento di Scienze Chimiche, Università di Padova, via Marzolo 1, 35131 Padova, Italy
${ }^{*}$ Dipartimento di Scienze Chimiche, Biologiche, Farmaceutiche ed Ambientali, Università di Messina, V.le F. Stagno d'Alcontres 31, 98166 Messina, Italy

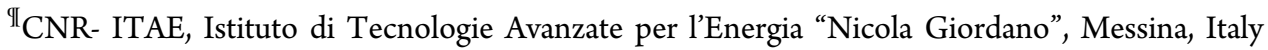

${ }^{\S}$ CNR-ISMN, Istituto per lo Studio dei Materiali Nanostrutturati, c/o Dipartimento di Scienze Chimiche, Biologiche, Farmaceutiche ad Ambientali, 98166, V.le F. Stagno D’Alcontres 31, Messina, Italy

"Dipartimento di Scienze Chimiche e Geologiche, Università di Modena e Reggio Emilia, via G. Campi 103, 41125 Modena, Italy

${ }^{\perp}$ CNR-ISMN, Istituto per lo Studio dei Materiali Nanostrutturati, c/o Dipartimento di Scienze Chimiche, Biologiche, Farmaceutiche ad Ambientali, 98166 V.le F. Stagno D’Alcontres 31, Messina, Italy
\end{abstract}

\section{Supporting Information}

ABSTRACT: In this work, we demonstrate the use of two-dimensional electronic spectroscopy (2DES) to study the mechanism and time scale of the femtosecond Stokes shift dynamics in molecules characterized by intramolecular charge transfer, such as distyryl-functionalized boron dipyrromethene (BODIPY) molecules. The obtained results demonstrate that 2DES allows clear and direct visualization of the phenomenon. The analysis of the $2 \mathrm{D}$ data in terms of $2 \mathrm{D}$ frequency-frequency decay associated maps provides indeed not only the time scale of the relaxation process but also the starting and the final point of the energy flow and the associated reorganization energy, identified by looking at the coordinates of a negative signature below the diagonal. The sensitivity of the 2DES technique to vibrational coherence dynamics also allowed the identification of a possible relaxation mechanism involving specific interaction between a vibrational mode of the dye and the solvent.

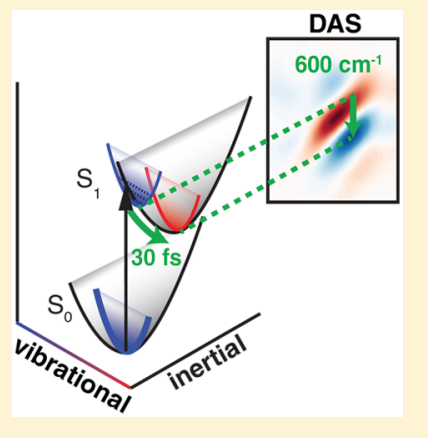

$\mathrm{T}$ he first steps of equilibration of excited states following photoexcitation in condensed phase have been the subject of intense investigation for many decades (see for example ref 1 for a recent review). Crucial in these ultrafast dynamics is the coupling between electronic and vibrational degrees of freedom of solute and solvent. In solution, indeed, the change of the dipole moment between ground and excited electronic states of the photoexcited solute is stabilized by reorganization of the solvent around the molecule and nuclei relaxation. This effect is spectroscopically revealed by the Stokes shift, i.e., the difference between the maximum energy of absorption and fluorescence. This shift often evolves in time after photoexcitation, witnessing a dynamic response to charge redistribution upon light absorption. As far as time scales are concerned, one distinguishes the so-called inertial response on a time scale of a few tens to a few hundreds of femtoseconds and the subsequent collective motion with diffusive character, typically developing on time scales from a few hundreds of femtoseconds to nanoseconds. ${ }^{2}$ The time development of the inertial response and the coupling mechanisms regulating at the molecular level these dynamics are key parameters in many fields, and in particular, for all those applications involving excitation energy conversions and dissipations, such as photosynthesis and artificial solar energy conversion. This topic is of particular interest nowadays, especially in light of recent developments suggesting a possible functional role of coherent dynamics in the inertial response time scales. ${ }^{3}$ Nonetheless, the characterization of the inertial component is particularly challenging because of its faster dynamics. Fluorescence up-conversion and 3PEPS measurements are typically exploited to this aim. ${ }^{4}$ However, while the former struggles to detect time scales faster than $40-50 \mathrm{fs},{ }^{5}$ the latter may require challenging interpretations when more than two

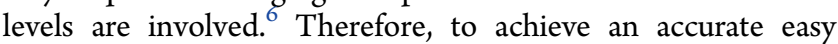
rationalization of the complex ultrafast dynamics of ICT molecules, novel approaches, as well as new spectroscopic tools, become essential. We have recently demonstrated that twodimensional electronic spectroscopy (2DES) can be conven-

Received: December 23, 2017

Accepted: February 15, 2018

Published: February 15, 2018 
(a)
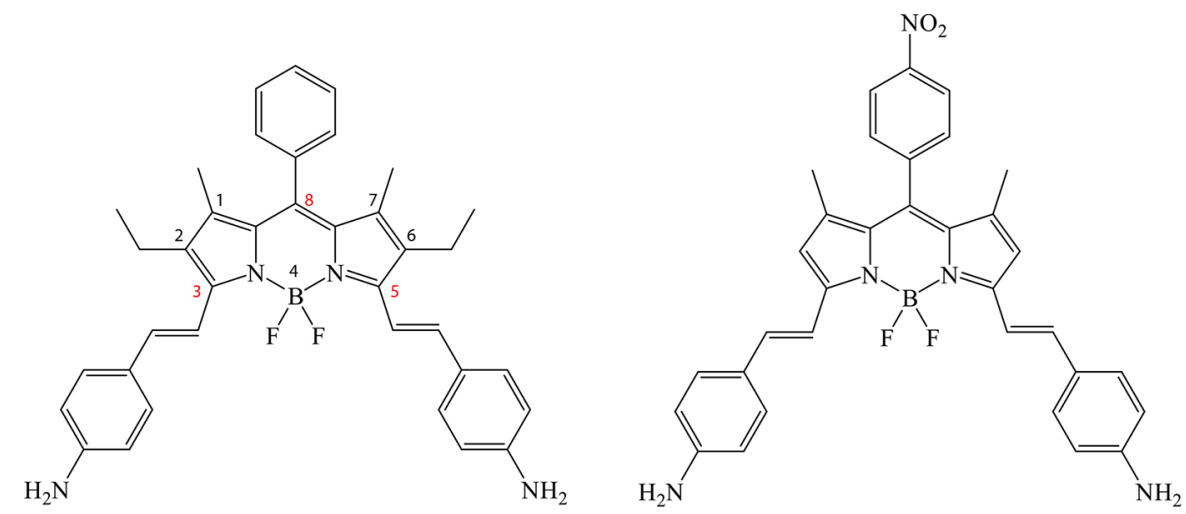

(b)

(c)
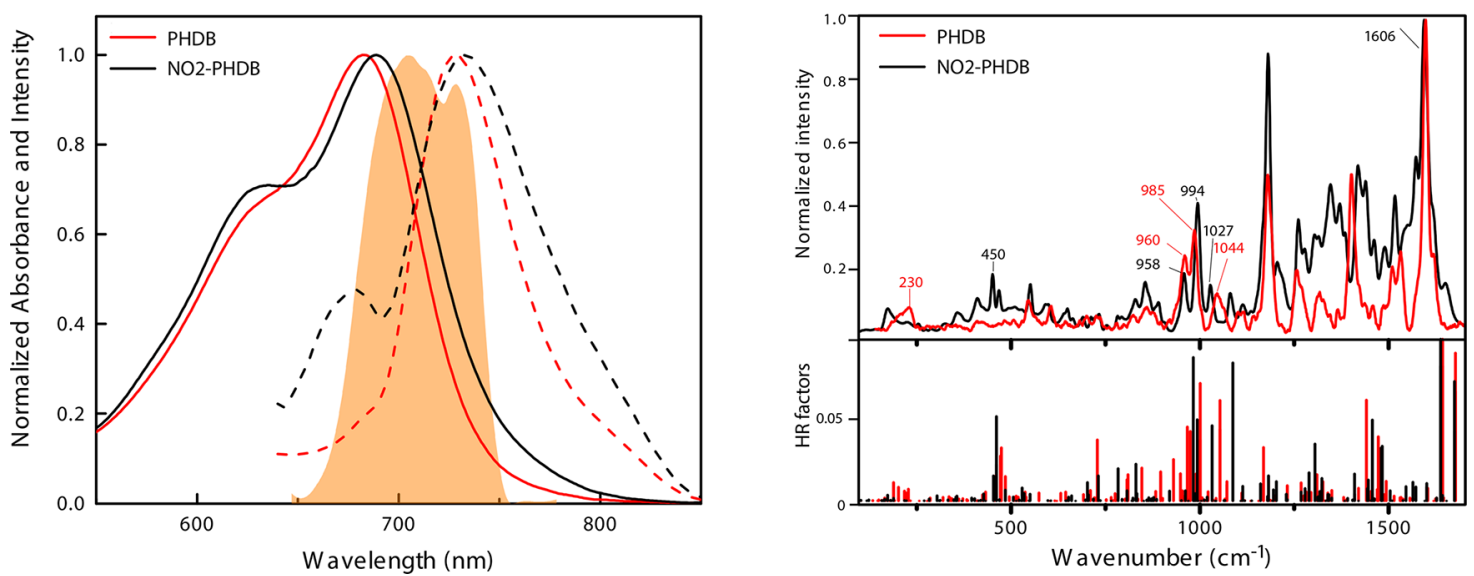

Figure 1. (a) Structures of NO2-PHDB and PHDB. (b) Absorption (solid lines) and emission (dashed lines) spectra of the molecules in THF solutions (red $=$ PHDB; black $=$ NO2-PHDB). The orange area represents the laser spectrum profile used in the 2DES experiments. $(\mathrm{c})$ Resonant Raman spectra of powders recorded with excitation at $633 \mathrm{~nm}$. The vibrational modes commented in the text (vide infra) are highlighted. Spectra have been normalized on the $1606 \mathrm{~cm}^{-1}$ band assigned to the $\mathrm{C}=\mathrm{C}-\mathrm{N}$ stretching. ${ }^{18}$ Computed stick Raman spectra computed at the harmonic level are also reported for comparison. The frequencies are scaled by a factor of 0.98 to account empirically for anharmonic effects.

iently exploited, together with newly developed data analysis tools, to reveal the fine ultrafast details of intraband relaxation dynamics with a time resolution up to $10 \mathrm{fs}^{8,9}$ Using a similar approach, here we exploit 2DES together with high-level timedependent density functional theory (TDDFT) calculations ${ }^{10}$ and novel data analysis tools to capture signatures of this process in a model molecular system.

The attention was focused in particular on polar molecules characterized by intramolecular charge transfer (ICT) because their pronounced difference of charge distribution in the ground and excited state makes these systems particularly suitable for following energy redistribution upon photoexcitation. Therefore, boron dipyrromethene (BODIPY) derivatives have been chosen. These molecules present highly promising chemical and photophysical properties. ${ }^{11-14}$ The charge distribution and the ICT character of BODIPY dyes can be finely tuned by introducing electron releasing or withdrawing groups at suitable positions. ${ }^{15,16}$ In particular, attachment of electron-donating styrylamino groups to a C8-phenyl substituted BODIPY core generates a push-pull structure resulting in pronounced spectral changes by activation of ICT and photoinduced electron transfer. ${ }^{17}$

In this work we focused our attention on two similar compounds: 8-phenyl-3,5-di(diamino)styryl-borondipyrromethene (PHDB) and the analogous 8-(p-nitrophenyl)-3,5di(diamino)styryl-borondipyrromethene (NO2-PHDB). The molecular structures are reported in Figure 1a. The choice of these particular structures has been motivated, beyond their recognized ICT character, by the possibility of using the molecules as building blocks for more complex supra-molecular structures.

The solvatochromic properties of amino distyryl-BODIPYs are already known and characterized; ${ }^{12,15}$ therefore, here we limited the analysis to only two different solvents, tetrahydrofuran (THF) and methanol $(\mathrm{MeOH})$, to assess the ICT character of the excited state and verify the effect of the nitro group. The photophysical properties of the two molecules in THF and $\mathrm{MeOH}$ solutions have been preliminarily characterized by steady-state absorption, fluorescence, and timeresolved fluorescence spectroscopy (see the Supporting Information). Figure $1 \mathrm{~b}$ reports the absorption and emission spectra of the two species in THF.

Also, the vibrational properties of the molecules have been fully characterized by recording the resonant Raman spectra on crystalline powders excited at $633 \mathrm{~nm}$ (Figure 1c). The Raman spectra of the two species are very similar, as expected considering the similarity of the backbone of the molecules. The main differences are recorded in the regions 400-500 and $1300-1500 \mathrm{~cm}^{-1}$ and can be ascribed to the contributions of the nitro group. ${ }^{19}$

The photophysical studies have also been supported by quantum mechanical (QM) calculations carried out at the DFT 
(a)

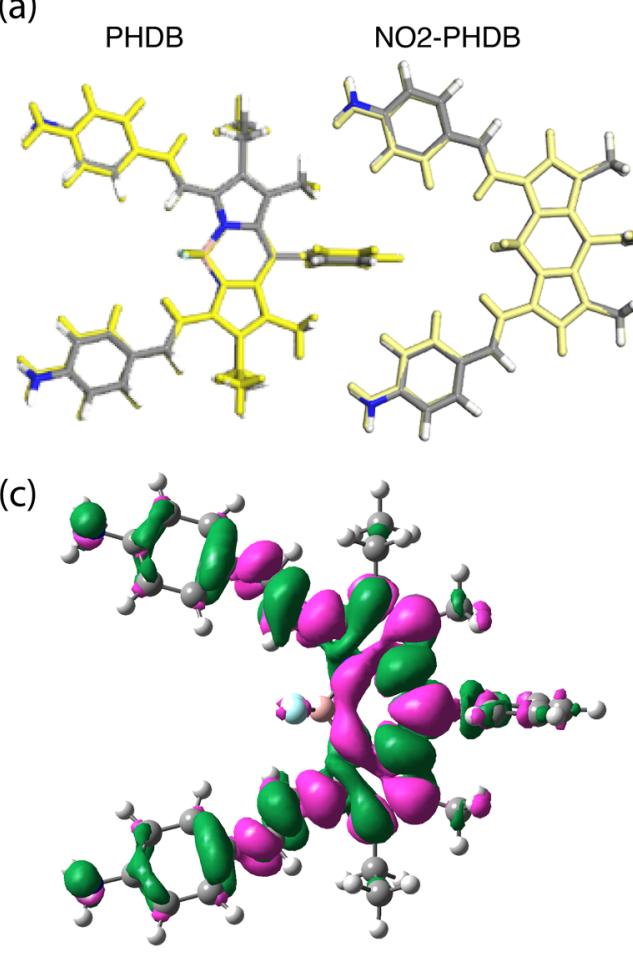

(b)

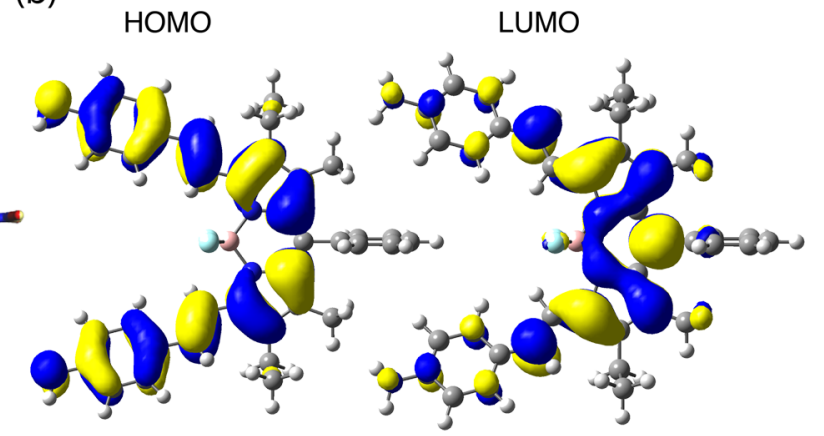

(d)

PHDB
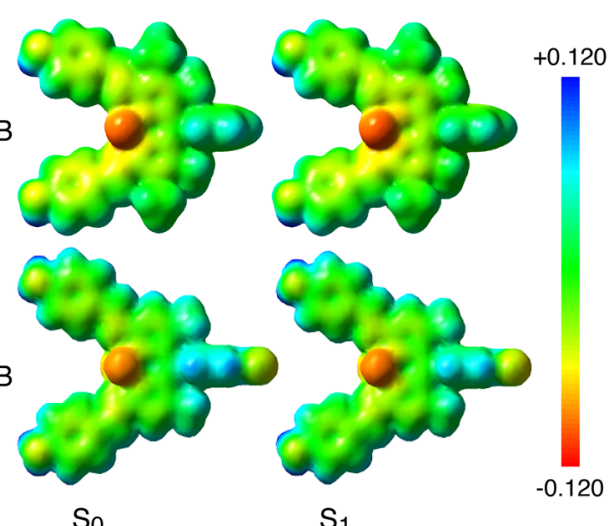

Figure 2. (a) Overlayered ground (sticks colored by element) and excited (yellow sticks) optimized structures of the two molecules investigated.(b) Kohn-Sham molecular orbitals involved during the $S_{0}-S_{1}$ transition for PHDB. (c) Electron density change $\left(\Delta \rho_{S_{1}-S_{0}}\right)$ upon absorption of light for PHDB. Dark green clouds represent the region where the electron density increases during the transition. (d) Electrostatic potential mapped onto the electronic density for PHDB and NO2-PHDB in THF solvent.

and TDDFT levels with the Gaussian09 suite of programs ${ }^{20}$ using the long-range corrected CAM-B3LYP ${ }^{21}$ functional coupled with the $6-311 \mathrm{G}(\mathrm{d}, \mathrm{p})$ basis set. In all the calculations the solvent effects were accounted for using the polarizable continuum model (PCM). ${ }^{22}$ The calculations of the groundand excited-state optimized structures highlight that no significant structural variations occur during the transition (Figure 2a). Figure 1c compares the simulated stick Raman spectra computed at the harmonic level with the experimental ones. A good agreement was found, especially in the prediction of the main differences between the spectra of the molecules in the region below $1000 \mathrm{~cm}^{-1}$. The assignment of the relevant normal modes is reported in Figure S2.2.

The calculations of vertical excitation and emission processes of the two molecules have been carried out by employing the nonequilibrium linear responses (NEQ-LR-PCM) and the equilibrium state specific (EQ-SS-PCM) approaches, respectively. ${ }^{23,24}$ All the values are reported in Table S2.1. They reveal that the first singlet excited state is due to a dipole-allowed $\pi \rightarrow$ $\pi^{*}$ transition with high oscillator strength from the highest occupied molecular orbital (HOMO) to the lowest unoccupied molecular orbital (LUMO) shown in Figure $2 \mathrm{~b}$ for PHDB. In both molecules, the HOMOs are delocalized in the whole molecule, whereas the LUMOs are mainly localized in the BODIPY core. The first electronic transition seems to have a partial ICT character as also observed by looking at the electron density change $\left(\Delta \rho_{\mathrm{S}_{1}-\mathrm{S}_{0}}\right)$ reported in Figure $2 \mathrm{c}$. To determine the spatial extent associated with the electronic transition and to better quantify its ICT character, the charge-transfer distance $\left(d_{\mathrm{CT}}\right)$ and the transferred charge $\left(q_{\mathrm{CT}}\right)$ have been calculated. ${ }^{25}$
The associated dipole $\left(\mu_{\mathrm{CT}}=q_{\mathrm{CT}} d_{\mathrm{CT}}\right)$ has then been determined as 4.70 and $4.97 \mathrm{D}$ for PHDB and NO2-PHDB in THF, respectively. Despite the presence of the electronwithdrawing nitro group, the calculations did not predict significant differences in the ICT character of the transition in the two molecules. The contour plots showing the electrostatic potential of the molecules (Figure $2 \mathrm{~d}$ ) confirm this similarity.

2D measurements have been performed on THF and $\mathrm{MeOH}$ solutions of PHDB and NO2-PHDB. The experimental setup has been already described in ref 26. More details about the experimental setup and the pulse properties are reported in the Supporting Information, section 4.1. Qualitatively, the 2D spectra obtained for the two molecules in the two analyzed solvents present similar features. Figure 3 summarizes only the results on THF solutions of NO2-PHDB, but the full series of data are available in the Supporting Information. Panel a illustrates the evolution of the rephasing $2 \mathrm{D}$ spectra at selected values of population time. In the investigated time range, the signal is dominated by a positive diagonal peak elongated along the diagonal direction, attributed to ground-state bleaching and stimulated emission of the $S_{1}$ state.

The dynamic evolution of the $2 \mathrm{D}$ maps has been studied with a recently proposed global analysis methodology. ${ }^{27}$ For all the samples, together with a long time constant ( $>500 \mathrm{fs}$ ) describing the overall decay of the maps, the dynamics in the investigated time window is characterized by a time constant of a few tens of femtoseconds (Table 1). The amplitude distribution of this short component can be shown in the form of 2D decay-associated spectrum (DAS), as exemplified in Figure $3 \mathrm{~b}$. Positive (negative) features in a DAS represent signals that are decaying (rising) with the associated time 
(a)
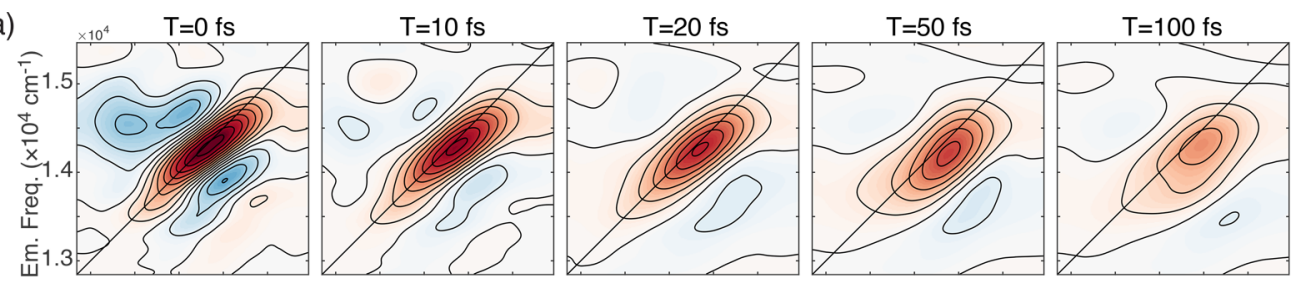

(c)

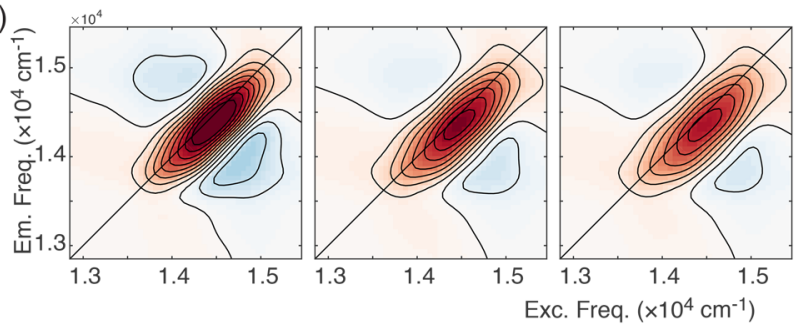

(e)

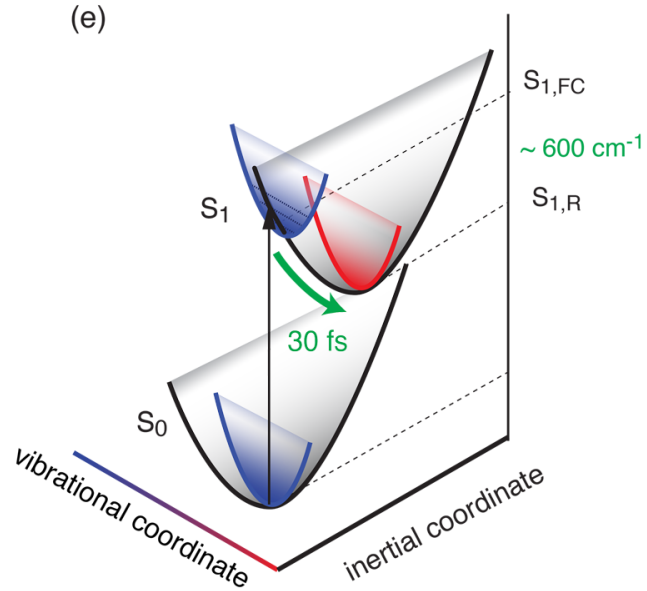

(f)

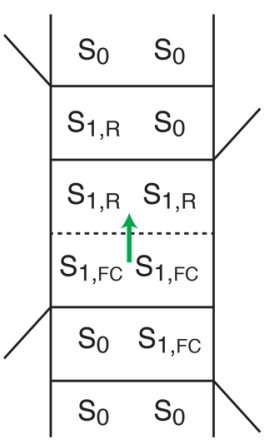

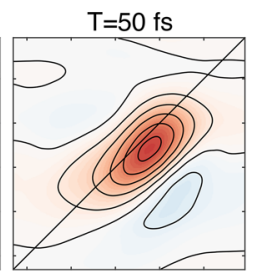

(b)

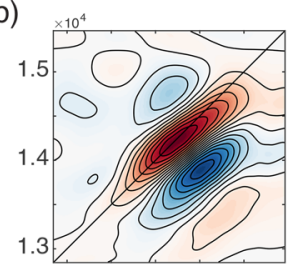

(d)

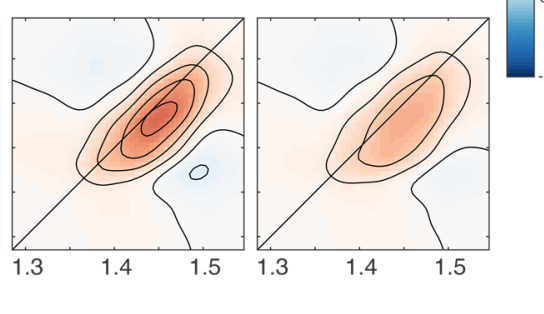

d)

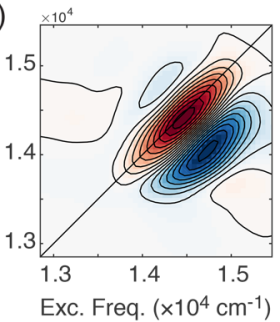

Figure 3. (a) $2 \mathrm{D}$ maps recorded for NO2-PHDB in THF at selected values of population time $T$. (b) $2 \mathrm{D}$ decay associated spectrum relative to the ultrafast relaxation dynamics with time constant $30 \mathrm{fs}$ (DAS1). (c and d) Same maps as obtained from the simulations. (e) Schematic illustration of the energy curves involved in the ultrafast relaxation dynamics after photoexcitation in the displaced harmonic oscillator model. Two orthogonal degrees of freedom are represented: the inertial coordinate (black) and the vibrational coordinates (blue for the FC coordinate and red for the relaxed $R$ coordinate). The green arrow represents the inertial $S_{1, \mathrm{FC}} \rightarrow S_{1, \mathrm{R}}$ relaxation, characterized by a time constant of $30 \mathrm{fs}$ for NO2-PHDB in THF. The reorganization energy for this process is estimated to be about $600 \mathrm{~cm}^{-1}$. (f) Feynman diagram describing the rising of the signal in the lower off-diagonal part of the 2D map and attributed to the $S_{1, \mathrm{FC}} \rightarrow S_{1, \mathrm{R}}$ relaxation. (g) Decay of the signal at FC state coordinates (14 500, 14500 $\mathrm{cm}^{-1}$; blue) and corresponding rising of the signal at relaxed coordinates $\left(14500,13800 \mathrm{~cm}^{-1}\right.$; red).

Table 1. Time Constants for the Inertial Relaxation Process Resulting from the Global Fitting Analysis for the Two Molecules in the Two Solvents

\begin{tabular}{lcc} 
& PHDB & NO2-PHDB \\
THF & $13 \mathrm{fs}$ & $29 \mathrm{fs}$ \\
$\mathrm{MeOH}$ & $16 \mathrm{fs}$ & $41 \mathrm{fs}$ \\
\hline
\end{tabular}

constant. The amplitude distribution of this DAS cannot be entirely justified with spectral diffusion, which would have given rise to symmetric negative signals above and below the diagonal. $^{27}$ Similarly to what was recently observed in a different system, ${ }^{9}$ a prominent negative feature below the diagonal witnesses a relaxation from higher to lower energy states. In the specific case of the molecules under investigation, this dynamics can be interpreted as the relaxation from the vertically excited Franck-Condon state (FC) toward a relaxed configuration, labeled with $\mathrm{R}$ in Figure 3e. The double-sided Feynmann diagram accounting for the signal in the lower diagonal region is illustrated in panel f of Figure 3, while panel $g$ shows the decay of the signal at FC coordinates (blue) and the corresponding rise, with the same time constant, of the signal at relaxed coordinates (red). This dynamics can, therefore, be interpreted as the previously defined inertial response of intraand intermolecular degrees of freedom.

Differently from the results of other techniques typically employed for the characterization of the inertial response, the DAS in Figure $3 \mathrm{~b}$ allows clear and direct visualization of the phenomenon, not only providing the time scale of the relaxation processes but also showing in a $2 \mathrm{D}$ frequencyfrequency correlation map (DAS) the starting (FC) and the final (R) point of the energy flow, identifiable with the $x$ and $y$ coordinate of the negative cross peak, respectively. One can thus readily estimate the reorganization energy involved in the dynamical process described by the specific time constant associated with the DAS.

In support of this approach, it is also possible to simulate the data and obtain these parameters from the simulation. In this specific case, the 2DES response was modeled using a two-level system coupled to an external bath. The interaction with the environment and nuclear degrees of freedom were incorporated into the line shape function associated with the electronic transition employing the Brownian oscillator formalism. ${ }^{28}$ 
(a)

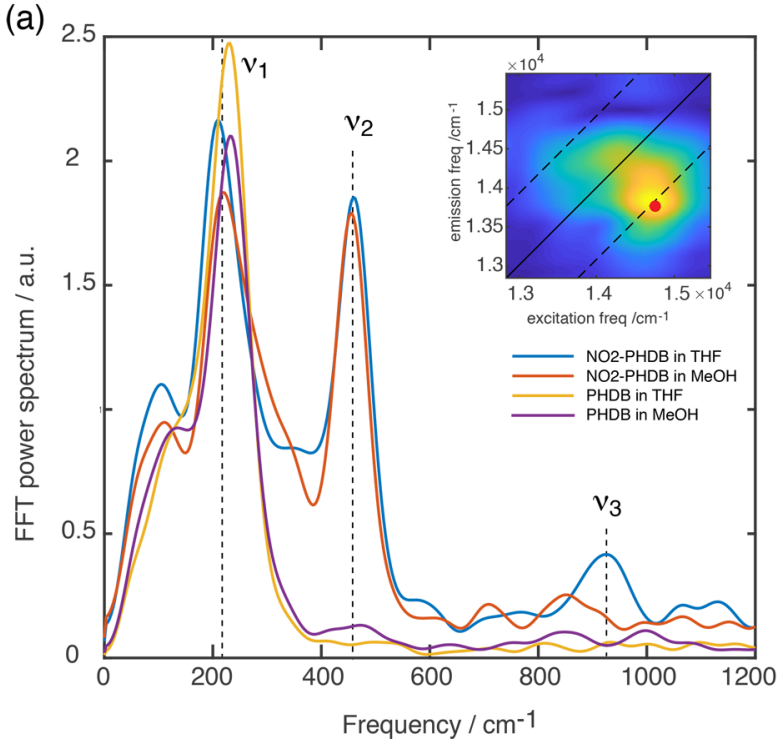

(b)

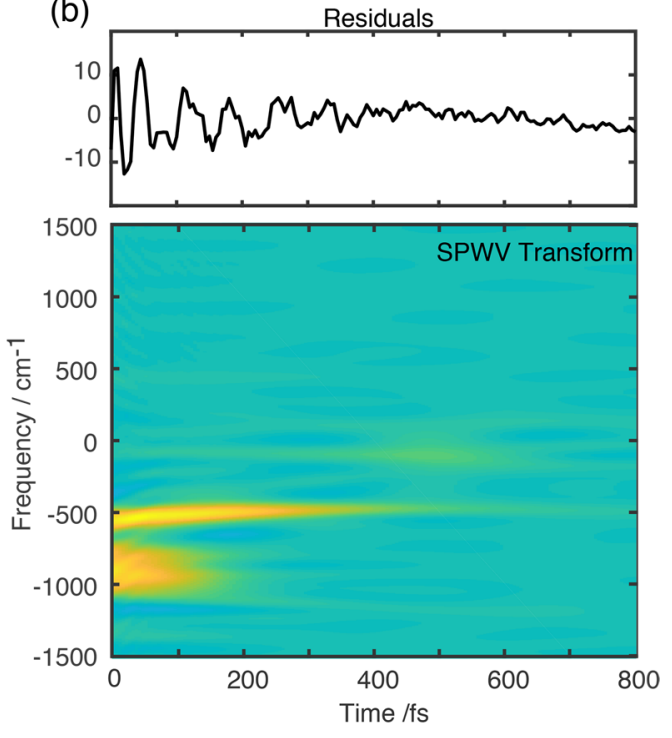

Figure 4. (a) Fourier spectra of the four samples highlighting the frequency components more strongly contributing to the beating pattern of the $2 \mathrm{D}$ signal. The spectra are obtained by Fourier transforming the integrated 2D maps. The main oscillating frequencies $\left(\nu_{1}=220, \nu_{2}=450\right.$, and $\nu_{3}=950$ $\mathrm{cm}^{-1}$ ) are emphasized. The inset shows the amplitude distribution of the $\nu_{3}$ beating mode for NO2-PHDB in THF. (b) Time-frequency transform analysis of the oscillating residuals for NO2-PHDB in THF at coordinates $(14500,13800) \mathrm{cm}^{-1}$ pinpointed with the red dot in the inset of panel a. Upper panel: oscillating residuals at the same coordinates. The feature at around $500 \mathrm{~cm}^{-1}$ is a residual of the $\nu_{2}$ signal, partially contributing also at these coordinates because of broadening effects.

Following standard nonlinear response function theory, ${ }^{29}$ the line shape function was then used as input to calculate the nonlinear response functions, which were fully convoluted with the laser pulse profile to obtain the 2DES spectra. To satisfactorily reproduce broadening and relaxation dynamics of the 2DES response, two overdamped Brownian oscillators were added to the line shape. A first oscillator accounts for the static inhomogeneous broadening, while the second introduces spectral diffusion and Stokes shift dynamics in the ultrafast time scale. A full agreement between experimental and simulated data, both in terms of shape of the 2D maps and amplitude distribution of the DAS, could be obtained imposing an ultrafast reorganization energy of 600 and $650 \mathrm{~cm}^{-1}$ for PHDB and NO2-PHDB in THF, respectively (Figure $3 c, d$ and the Supporting Information).

A careful look at the time constants characterizing the inertial relaxation of the two molecules in the two solvents reveals slightly different rates, as summarized in Table 1 . The values found for the $\mathrm{MeOH}$ solutions are in reasonably good agreement with recent measurements on a molecule with a similar structure. ${ }^{30}$ These time constants are very fast and, especially in the case of PHDB, close to the time resolution of the experiment, leading to a non-negligible uncertainty in their determination. Nevertheless, beyond the exact value of time constants, the comparison of the decays recorded for different sample solutions reveals trends as a function of solvent and presence of the nitro group. In general, the dynamics in THF always results in faster decay than in $\mathrm{MeOH}$. This trend can be justified in light of the higher polarizability of $\mathrm{THF}^{31}$ which is able to reorganize faster toward the new equilibrium configuration. On the other hand, the functionalization with the nitro group has the effect of slowing the inertial relaxation. This effect could be connected with the more significant spreading of the charge distribution in the NO2-PHDB compound (Figure 2d), which is expected to slow the inertial response.
Additional details about the degrees of freedom more actively involved in the inertial relaxation can be obtained looking at the evolution of vibrational coherent wave packets. The initial photoexcitation can generate coherent superpositions of vibrational levels, often leading to coherent wave packet dynamics in the excited-state potential manifold, whose dephasing strongly depends on the properties of the potential energy surface and the nature of the interaction with the environment.

The evolution of vibrational coherent wave packet is manifested in 2D spectra as oscillations of the signal along the population time. The corresponding beating frequencies are typically extracted by performing a Fourier transform (FT) of the oscillating residuals. The FT spectra calculated by integrating the whole 2D maps for the four samples (Figure 4a) show that the oscillations in the $2 \mathrm{D}$ signal of PHDB are dominated by a mode beating at $\nu_{1}=230 \mathrm{~cm}^{-1}$, both in THF and $\mathrm{MeOH}$. The oscillating pattern of NO2-PHDB presents more fetaures: in $\mathrm{MeOH}$, together with a signal at $\nu_{1}=220$ $\mathrm{cm}^{-1}$, a strong signal at $\nu_{2}=450 \mathrm{~cm}^{-1}$ also emerges. In THF, an additional broad feature centered at $\nu_{3}=950$ appears. All these frequencies roughly correspond to vibrational modes also detected in the Raman spectra and identified in the calculations (Figure 1c). The presence of a higher number of frequencies in the oscillating pattern of NO2-PHDB follows the trend already recognized in Raman spectra, where the presence of additional signals at about $450 \mathrm{~cm}^{-1}$ was previously identified. For both molecules in both solvents, the $\nu_{1}$ and $\nu_{2}$ signals are characterized by a dephasing time longer than the investigated time window, which is therefore estimated to be $>0.5$ ps. Furthermore, the amplitude distribution of these modes across the $2 \mathrm{D}$ maps follows the behavior expected for vibrational coherence, ${ }^{28}$ supporting their interpretation in terms of vibrational modes of the molecules.

Different is the behavior of $\nu_{3}$, showing the maximum amplitude in the region below diagonal, in correspondence of 
the negative cross peak feature localized in the DAS (inset of Figure $4 a$ ) and characterized by a much shorter dephasing time. Figure $4 \mathrm{~b}$ shows the results of a time-frequency transform (TFT) applied to the oscillating residuals recorded at coordinates $(14500,13800) \mathrm{cm}^{-1}$ in THF. This analysis overcomes the limitations of conventional methods based on Fourier transforms maintaining both frequency and time resolution. ${ }^{32}$ The figure immediately highlights that the signal at $950 \mathrm{~cm}^{-1}$ is characterized by a considerably shorter damping time and, in agreement with the time-frequency indetermination principle, by a larger bandwidth. The fast dephasing time of this signal suggests the presence of additional dynamic mechanisms contributing to the evolution of this vibrational coherence. A first indication to identify this mechanism is that, as shown in the amplitude distribution map shown in the inset of Figure $4 a$, this frequency contributes mainly at off-diagonal coordinates where a signal due to the inertial environment reorganization has been already ascertained.

THF solvent has a vibrational mode with a similar frequency at $914 \mathrm{~cm}^{-1}$. However, the assignment of this component to a nonresonant signal of the pure solvent can be ruled out considering that it does not appear in THF solutions of PHDB and that its amplitude distribution and dynamics do not follow the expected behavior. ${ }^{33,34}$

A careful look at the 2D response of NO2-PHDB in $\mathrm{MeOH}$ reveals the possible presence of a weak signal at slightly higher frequency (about $1050 \mathrm{~cm}^{-1}$ ). This mode is not recognizable from the integrated Fourier spectra in Figure 4a, but it weakly emerges from the analysis of the residuals at a single coordinate (14500, $13800 \mathrm{~cm}^{-1}$ ). The corresponding TFT analysis reported in Figure S4.2 reveals a time behavior similar to the one already found for $\nu_{3}$ in THF, although characterized by a slightly longer dephasing time. It is noteworthy that, also in this case, a solvent vibration close in frequency is present $(\mathrm{MeOH}$ has a vibrational mode at $1040 \mathrm{~cm}^{-1}$ ).

All these pieces of evidence seem to suggest that the $\nu_{3}$ signal is strongly affected by a specific interaction of the NO2-PHDB molecule with the THF solvent, likely enhanced by the resonance between vibronic energy gaps of dye and solvent molecules. This phenomenon is more evident in THF than in $\mathrm{MeOH}$. This can be justified by recognizing that the NO2PHDB molecule has two intense vibrational modes (at 958 and $994 \mathrm{~cm}^{-1}$ ) near resonant with the vibrational mode of THF at $914 \mathrm{~cm}^{-1}$, whereas there is only a weak mode at $1027 \mathrm{~cm}^{-1}$ that can be resonant with the vibrational mode of $\mathrm{MeOH}$ at 1040 $\mathrm{cm}^{-1}$. The absence of distinguishable features at $\nu_{3}$ in PHDB can instead be justified considering three main aspects: (i) the overwhelming intensity of the $\nu_{1}$ signal, dominating the oscillating response and possibly covering weaker contributions; (ii) the Raman modes of PHDB in the region 900-1000 $\mathrm{cm}^{-1}$ are characterized by a slightly smaller intensity with respect to the nitro-analogous; (iii) the faster relaxation dynamics of PHDB (see Table 1), probably too fast to guarantee the activation of the coupling mechanism.

Because the $\nu_{3}$ beating mode is dampened in the same time scale found for the inertial environment reorganization process, it is tempting to associate this beating dynamics to the same process. In particular, we could suppose that the presence of a vibrational mode of the solvent, resonant with a vibrational mode of the dye, acts as a sort of sink for the dye wave packets, causing a fast dephasing of corresponding vibrations and promoting the relaxation toward the $\mathrm{R}$ coordinates. This mechanism, active for NO2-PHDB more in THF than in
$\mathrm{MeOH}$, could also contribute to explain the faster relaxation recorded in the former solvent. This would imply that the inertial relaxation from $\mathrm{FC}$ to $\mathrm{R}$ state can be strongly influenced by vibrational modes of the solvent, and it would open a completely new view into the microscopic details affecting the initial ultrafast relaxation dynamics.

The idea of a vibrational energy flow from one mode to another inside a molecule and from one molecule to another is certainly not new. Vibrational energy transfer from solute to solvent has been the subject of intense investigation in the last two decades, ${ }^{2,35}$ but the experimental observations are typically limited to spectroscopies in the IR regime and thus in the electronic ground state. $^{36,37}$ Our 2DES data seem instead to provide new evidence for a strong coupling mechanism actively contributing to the ultrafast relaxation dynamics in the excited state of the solute. Clearly, to verify the generality of the process and clarify the details of the interplay between solvent and dye degrees of freedom in the ultrafast inertial relaxation dynamics more systematic investigations on different solvents and molecules are required.

In conclusion, this work demonstrates that 2DES measurements complemented by suitably developed data analysis procedures and TDDFT calculations represent an outstanding tool to characterize the ultrafast dynamic steps of inertial equilibration dynamics in ICT molecules. The analysis of the $2 \mathrm{D}$ data in terms of $2 \mathrm{D}$ frequency-frequency decay associated maps not only provides the time constant of the relaxation process but also allows a direct determination of the associated reorganization energy, identifiable by looking at the coordinates of a negative signature below the diagonal. The sensitivity of the $2 \mathrm{DES}$ technique to vibrational coherence dynamics also allowed the identification of a possible relaxation mechanism involving specific interaction between a vibrational mode of the dye and the solvent. While further measurements and theoretical investigations are surely needed to verify the actual nature of this mechanism, several experimental pieces of evidence point toward this interpretation and suggest an intriguing role of the solvent in the relaxation dynamics of ICT molecules. Owing to the importance and diffusion of ICT states in biology and materials science, recognizing their characteristic features in the 2DES response starting from the study of model molecules is particularly relevant.

\section{ASSOCIATED CONTENT}

\section{S Supporting Information}

The Supporting Information is available free of charge on the ACS Publications website at DOI: 10.1021/acs.jpclett.7b03393.

Additional linear characterization and 2DES data; computational details (PDF)

\section{AUTHOR INFORMATION}

\section{Corresponding Author}

*E-mail: elisabetta.collini@unipd.it. ORCID

Luca Bolzonello: 0000-0003-0893-5743

Annalisa Polo: 0000-0001-5724-2607

Alfonso Pedone: 0000-0003-3772-7222

Maria Angela Castriciano: 0000-0002-1514-8820

Elisabetta Collini: 0000-0002-1019-9100

Notes

The authors declare no competing financial interest. 


\section{ACKNOWLEDGMENTS}

This work is financially supported by the ERC Starting Grant QUENTRHEL (278560) and PRIN-MIUR 2015 (Prot. n.2015XBZ5YA).

\section{REFERENCES}

(1) Kumpulainen, T.; Lang, B.; Rosspeintner, A.; Vauthey, E. Ultrafast Elementary Photochemical Processes of Organic Molecules in Liquid Solution. Chem. Rev. 2017, 117, 10826-10939.

(2) Bagchi, B.; Jana, B. Solvation Dynamics in Dipolar Liquids. Chem. Soc. Rev. 2010, 39, 1936.

(3) Scholes, G. D.; Fleming, G. R.; Chen, L. X.; Aspuru-Guzik, A.; Buchleitner, A.; Coker, D. F.; Engel, G. S.; van Grondelle, R.; Ishizaki, A.; Jonas, D. M.; et al. Using Coherence to Enhance Function in Chemical and Biophysical Systems. Nature 2017, 543, 647-656.

(4) Fleming, G. R.; Cho, M. Chromophore-Solvent Dynamics. Annu. Rev. Phys. Chem. 1996, 47, 109-134.

(5) Eom, I.; Joo, T. Polar Solvation Dynamics of Coumarin 153 by Ultrafast Time-Resolved Fluorescence. J. Chem. Phys. 2009, 131, 244507.

(6) Fleming, G. R.; Passino, S. A.; Nagasawa, Y. The Interaction of Solutes with Their Environments. Philos. Trans. R. Soc., A 1998, 356, 389-404.

(7) Jumper, C. C.; Arpin, P. C.; Turner, D. B.; McClure, S. D.; Rafiq, S.; Dean, J. C.; Cina, J. A.; Kovac, P. A.; Mirkovic, T.; Scholes, G. D. Broad-Band Pump-Probe Spectroscopy Quantifies Ultrafast Solvation Dynamics of Proteins and Molecules. J. Phys. Chem. Lett. 2016, 7, 4722-4731.

(8) Meneghin, E.; Leonardo, C.; Volpato, A.; Bolzonello, L.; Collini, E. Mechanistic Insight into Internal Conversion Process within QBands of Chlorophyll a. Sci. Rep. 2017, 7, 11389.

(9) Bolzonello, L.; Fassioli, F.; Collini, E. Correlated Fluctuations and Intraband Dynamics of J-Aggregates Revealed by Combination of 2DES Schemes. J. Phys. Chem. Lett. 2016, 7, 4996-5001.

(10) Runge, E.; Gross, E. K. U. Density-Functional Theory for TimeDependent Systems. Phys. Rev. Lett. 1984, 52, 997-1000.

(11) Ulrich, G.; Ziessel, R.; Harriman, A. The Chemistry of Fluorescent Bodipy Dyes: Versatility Unsurpassed. Angew. Chem., Int. Ed. 2008, 47, 1184-1201.

(12) Lu, H.; Mack, J.; Yang, Y.; Shen, Z. Structural Modification Strategies for the Rational Design of Red/NIR Region BODIPYs. Chem. Soc. Rev. 2014, 43, 4778-4823.

(13) Ziessel, R.; Ulrich, G.; Harriman, A. The Chemistry of Bodipy: A new El Dorado for Fluorescence Tools. New J. Chem. 2007, 31, 496.

(14) Boens, N.; Leen, V.; Dehaen, W. Fluorescent Indicators Based on BODIPY. Chem. Soc. Rev. 2012, 41, 1130-1172.

(15) Huang, L.; Zhao, J. Iodo-Bodipys as Visible-Light-Absorbing Dual-Functional Photoredox Catalysts for Preparation of Highly Functionalized Organic Compounds by Formation of C-C Bonds via Reductive and Oxidative Quenching Catalytic Mechanisms. RSC Adv. 2013, 3, 23377.

(16) Bozdemir, O. A.; Guliyev, R.; Buyukcakir, O.; Selcuk, S.; Kolemen, S.; Gulseren, G.; Nalbantoglu, T.; Boyaci, H.; Akkaya, E. U. Selective Manipulation of ICT and PET Processes in Styryl-Bodipy Derivatives: Applications in Molecular Logic and Fluorescence Sensing of Metal Ions. J. Am. Chem. Soc. 2010, 132, 8029-8036.

(17) Ni, Y.; Wu, J. Far-red and Near Infrared BODIPY Dyes: Synthesis and Applications for Fluorescent $\mathrm{pH}$ Probes and BioImaging. Org. Biomol. Chem. 2014, 12, 3774.

(18) Adarsh, N.; Ramya, A. N.; Maiti, K. K.; Ramaiah, D. Unveiling NIR Aza-Boron-Dipyrromethene (BODIPY) Dyes as Raman Probes: Surface-Enhanced Raman Scattering (SERS)-Guided Selective Detection and Imaging of Human Cancer Cells. Chem. - Eur. J. 2017, 23, 14286-14291.

(19) Passingham, C.; Hendra, P.; et al. The Raman Spectra of Some Aromatic Nitro Compounds. Spectrochim. Acta Part A Mol. Spectrosc. Mol. Spectrosc. 1991, 47, 1235-1245.
(20) Frisch, M. J.; Trucks, G.; Schlegel, H. B.; Scuseria, G. E.; Robb, M. A.; Cheeseman, J. R.; Scalmani, G.; Barone, V.; Mennucci, B.; Petersson, G. A. et al. Gaussian 09; Gaussian, Inc.: Wallingford, CT, 2009.

(21) Yanai, T.; Tew, D. P.; Handy, N. C. A New Hybrid ExchangeCorrelation Functional Using the Coulomb-Attenuating Method (CAM-B3LYP). Chem. Phys. Lett. 2004, 393, 51-57.

(22) Cancès, E.; Mennucci, B.; Tomasi, J. A New Integral Equation Formalism for the Polarizable Continuum Model: Theoretical Background and Applications to Isotropic and Anisotropic Dielectrics. J. Chem. Phys. 1997, 107, 3032-3041.

(23) Improta, R.; Scalmani, G.; Frisch, M. J.; Barone, V. Toward Effective and Reliable Fluorescence Energies in Solution by a New State Specific Polarizable Continuum Model Time Dependent Density Functional Theory Approach. J. Chem. Phys. 2007, 127, 074504.

(24) Improta, R.; Barone, V.; Scalmani, G.; Frisch, M. J. A StateSpecific Polarizable Continuum Model Time Dependent Density Functional Theory Method for Excited State Calculations in Solution. J. Chem. Phys. 2006, 125, 054103.

(25) Adamo, C.; Le Bahers, T.; Savarese, M.; Wilbraham, L.; García, G.; Fukuda, R.; Ehara, M.; Rega, N.; Ciofini, I. Exploring Excited States Using Time Dependent Density Functional Theory and Density-Based Indexes. Coord. Chem. Rev. 2015, 304-305, 166-178.

(26) Bolzonello, L.; Volpato, A.; Meneghin, E.; Collini, E. Versatile Setup for High-Quality Rephasing, Non-Rephasing, and Double Quantum 2D Electronic Spectroscopy. J. Opt. Soc. Am. B 2017, 34, 1223.

(27) Volpato, A.; Bolzonello, L.; Meneghin, E.; Collini, E. Global Analysis of Coherence and Population Dynamics in 2D Electronic Spectroscopy. Opt. Express 2016, 24, 24773-24785.

(28) Butkus, V.; Valkunas, L.; Abramavicius, D. Molecular VibrationsInduced Quantum Beats in Two-Dimensional Electronic Spectroscopy. J. Chem. Phys. 2012, 137, 044513.

(29) Mukamel, S. Principles of Nonlinear Optical Spectroscopy; Oxford University Press: Oxford, U.K., 1995.

(30) Lee, Y.; Das, S.; Malamakal, R. M.; Meloni, S.; Chenoweth, D. M.; Anna, J. M. Ultrafast Solvation Dynamics and Vibrational Coherences of Halogenated Boron-Dipyrromethene Derivatives Revealed through Two-Dimensional Electronic Spectroscopy. J. Am. Chem. Soc. 2017, 139, 14733-14742.

(31) Bosque, R.; Sales, J. Polarizabilities of Solvents from the Chemical Ccomposition. J. Chem. Inf. Comput. Sci. 2002, 42, 11541163.

(32) Volpato, A.; Collini, E. Time-frequency Methods for Coherent Spectroscopy. Opt. Express 2015, 23, 20040.

(33) Fransted, K. A.; Caram, J. R.; Hayes, D.; Engel, G. S. TwoDimensional Electronic Spectroscopy of Bacteriochlorophyll a in Solution: Elucidating the Coherence Dynamics of the FennaMatthews-Olson Complex Using its Chromophore as a Control. J. Chem. Phys. 2012, 137, 125101.

(34) Hunt, N. T.; Jaye, A. A.; Meech, S. R. Ultrafast Dynamics in Complex Fluids Observed through the Ultrafast Optically-Heterodyne-Detected Optical-Kerr-Effect (OHD-OKE). Phys. Chem. Chem. Phys. 2007, 9, 2167.

(35) Kenkre, V. M.; Tokmakoff, A.; Fayer, M. D. Theory of Vibrational Relaxation of Polyatomic Molecules in Liquids. J. Chem. Phys. 1994, 101, 10618-10629.

(36) Elsaesser, T. Two-Dimensional Infrared Spectroscopy of Intermolecular Hydrogen Bonds in the Condensed Phase. Acc. Chem. Res. 2009, 42, 1220-1228.

(37) Chuntonov, L. 2D-IR Spectroscopy of Hydrogen-BondMediated Vibrational Excitation Transfer. Phys. Chem. Chem. Phys. 2016, 18, 13852-13860. 